\section{Soutenir la motivation des} participants aux M0OC: quels rôles pour la ludification, la mobilité et l'aspect social?
Thierry KARSENTI

Université de Montréal thierry.karsenti@umontreal.ca

Julien BUGMANN

Université de Montréal julien.bugmann@umontreal.ca

\title{
Sustaining the motivation of $\mathrm{MOOC}$ partici- pants: What are the effects of gamification, mobility, and the social dimension?
}

\section{Recherche scientifique avec données empiriques}

\section{Résumé}

Les objectifs de nos travaux étaient de décrire le rôle de la ludification des apprentissages et de la mobilité (apprentissage nomade) dans la motivation des participants à un MOOC, et d'établir d'autres caractéristiques d'un MOOC susceptibles de participer à la motivation des apprenants. Au moyen de deux enquêtes, nous avons interrogé quelque 4669 participants d'Afrique. Les données recueillies permettent d'abord de brosser un portrait général des principaux avantages et défis rencontrés par les participants à ce MOOC. Nous nous sommes ensuite intéressés à l'impact du MOOC sur la motivation des apprenants. Nous avons cherché à comprendre le rôle des stratégies de ludification mises en place, de même que l'intérêt des apprenants pour les questions d'apprentissage mobile ou nomade dans un MOOC. Nos résultats révèlent notamment que les caractéristiques mises en place dans la structure du MOOC CERTICE participent largement à la motivation des participants. Par ailleurs, les aspects mobiles semblent eux aussi très recherchés par ces derniers.

\section{Mots clés}

MOOC, formation à distance, motivation, apprentissage nomade, ludification

\begin{abstract}
This study aimed to describe how students enrolled in a MOOC can be motivated by gamification, mobile learning modes (nomadic learning), and other MOOC features. We conducted two surveys in a total sample of 4,669 African students. Based on their responses, we paint a broad portrait of the main benefits and challenges as perceived by the students. We then look at how the MOOC impacts student motivation. We wanted to shed light on how current gamification strategies as well as mobile learning modes can encourage students to continue taking a MOOC. The results show that certain features of the MOOC CERTICE program boost student motivation. Mobile capabilities also appear to be highly popular with students.
\end{abstract}

\section{Keywords}

MOOC, distance learning, motivation, mobile learning, gamification 


\section{Contexte}

On peut considérer les MOOC (massive open online courses) comme une nouvelle forme d'éducation à distance dont la popularité dans les universités peut être qualifiée de phénoménale ces dernières années (voir notamment le numéro spécial de la RITPU : Karsenti, Depover, Komis et Dumouchel, 2015). En effet, depuis 2011, plusieurs des plus grandes universités du monde ont participé sans relâche à cette nouvelle forme de ruée vers l'or, qui affiche des chiffres parfois troublants. Par exemple,

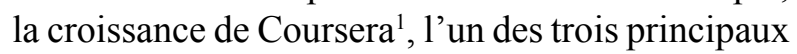
acteurs des MOOC avec Udacity ${ }^{2}$ et edX ${ }^{3}$, aura été plus rapide que celle de Facebook, YouTube ou Twitter, avec près de six millions «d'apprenants » en moins de deux ans. Quant à Udacity, il détient toujours le record du nombre de participants dans un MOOC, soit 300 000, pour son cours d'introduction à l'informatique. Signe de ce succès majeur, près de 40 millions de personnes issues de plus de 250 pays sont actuellement inscrites à un MOOC, et ce nombre continue de croître année après année (Karsenti, 2013, 2015; Karsenti et al., 2015). En outre, comme le fait remarquer Achard (2016), même si ce phénomène touche avant tout l'enseignement postsecondaire, il est de plus en plus associé à de nouvelles formes de recherche telles que les sciences citoyennes ${ }^{4}$.

Néanmoins, la popularité remarquable des MOOC ne leur permet pas pour autant d'éviter de faire face à de nombreux défis relevés depuis plusieurs années dans les formations à distance. Parmi les plus importants, on retrouve d'abord le faible taux de réussite (Breslow et al., 2013; Gillani, 2013; Karsenti, 2013). Bon nombre d'études montrent que les taux de réussite à un MOOC - lorsqu'ils sont révélés, ce qui n'est pas toujours le cas - sont singulièrement faibles puisque souvent, moins de $5 \%$ des participants terminent la formation. Ho et al. (2014) révélaient d'ailleurs que sur les 841687 étu-

$\begin{array}{ll}1 & \text { http://coursera.org } \\ 2 & \text { http://udacity.com } \\ 3 & \text { http://edx.com } \\ 4 & \text { http://www.cnrs.fr/comets/spip.php?article125 }\end{array}$

diants inscrits à 17 MOOC à l'Université Harvard et au MIT, seulement 43196 avaient terminé, pour un taux de réussite de $5,1 \%$. Ces faibles taux ont même amené plusieurs chercheurs à les présenter comme étant catastrophiques (Konnikova, 2014) ou encore à qualifier les participants à un MOOC de « touristes des formations à distance » (Xiong et al., 2015). Pour plusieurs chercheurs, c'est la motivation des apprenants qui expliquerait le mieux ce faible taux de réussite (Acosta, Otero et Valderrama, 2014; Alraimi, Zo et Ciganek, 2015; Bulger, Bright et Cobo, 2015; Chang, Hung et Lin, 2015; Freitas, Morgan et Gibson, 2015; García Espinosa, Tenorio Sepúlveda et Ramirez Montoya, 2015; Goldberg et al., 2015; Gray, 2015; Greene, Oswald et Pomerantz, 2015; Hew et Cheung, 2014; Jordan, 2015; Kizilcec et Schneider, 2015; Xiong et al., 2015).

Si l'on souhaite que les MOOC soient susceptibles d'adhérer à l'idéal d'une université humboldtienne 2.0 (Flückiger et Achard, 2016), il semble donc nécessaire de trouver des pistes de solutions au problème de motivation des apprenants participant à un MOOC. C'est ce à quoi aspire cette étude dont le premier objectif est de décrire le rôle de la ludification des apprentissages et de la mobilité (apprentissage nomade) dans la motivation de participants au MOOC CERTICE (voir section suivante). Le second objectif de cette étude est d'établir d'autres caractéristiques d'un MOOC susceptibles de participer à la motivation des apprenants.

\section{Le MOOC CERTICE de I'AUF}

En 2015, l'Agence universitaire de la Francophonie (AUF), en partenariat avec l'Organisation des Nations Unies pour l'éducation, la science et la culture (UNESCO) et le Réseau international francophone des établissements de formation de formateurs (RIFEFF), lançait le MOOC CERTICE (Certification des compétences TICE des enseignants). Le MOOC CERTICE propose deux variantes qui sont CERTICEscol et CERTICEsup. La première vise à former puis à certifier les enseignants du primaire et du secondaire et leurs encadreurs aux usages éducatifs des technologies de l'information et

2016 - Revue internationale des technologies en pédagogie universitaire, 13(2-3) 
de la communication pour l'éducation (TICE), alors que la seconde a pour objectif d'apporter les ressources nécessaires à la certification de doctorants, enseignants et formateurs du supérieur à l'utilisation des TICE. Notre étude porte ici uniquement sur la première variante, CERTICEscol. Ce dispositif de formation s'appuie principalement sur un référentiel élaboré par l'UNESCO (aussi appelé continuum des approches de l'UNESCO) qui se décline en six domaines (figure 1). Ce référentiel définit les compétences TIC que les enseignants doivent eux-mêmes posséder afin de pouvoir les transmettre à leurs élèves.

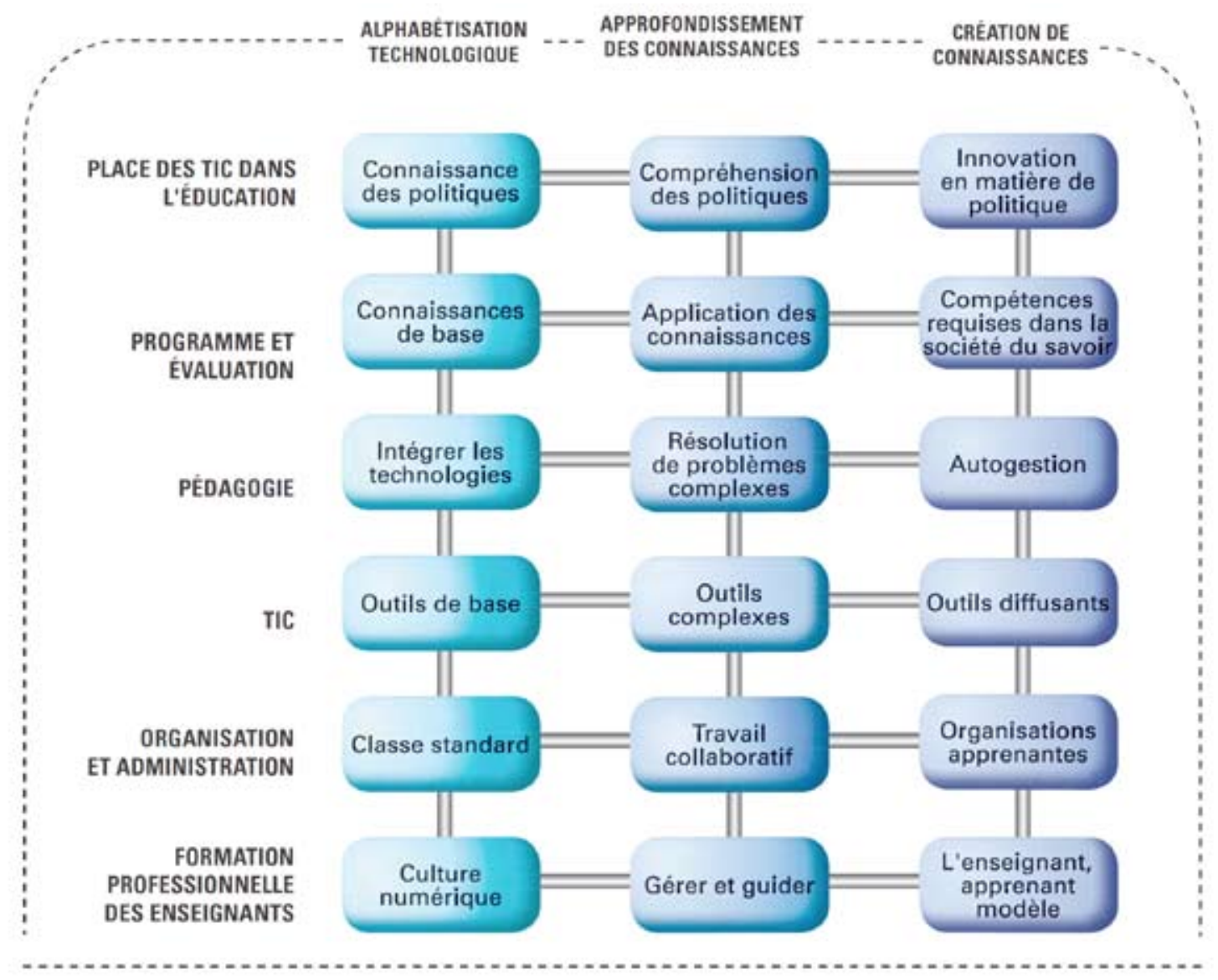

Figure 1: Graphique représentant les 6 domaines du référentiel élaboré par I'UNESCO

Ce MOOC est gratuit et dure de six à neuf semaines selon la version suivie (niveau 1 ou niveau 2). Les enseignants peuvent obtenir une « attestation de suivi » gratuite, après avoir satisfait aux exigen-

ces du MOOC, mais aussi opter pour l'inscription à une certification payante amenant à une reconnaissance académique par la délivrance de crédits universitaires.

La pertinence sociale et scientifique de cette re- 
cherche semble particulièrement importante quand on connait les besoins en formation en Afrique, tout particulièrement dans le domaine du numérique en éducation. Il s'agit d'un domaine où peu d'écoles de formation des maîtres possèdent les ressources ou les compétences requises pour proposer une telle formation à leurs étudiants. Le MOOC CERTICE de l'AUF serait donc susceptible de jouer un rôle capital dans la formation des enseignants francophones de l'Afrique pour l'usage du numérique en éducation. Mais pour cela, encore faut-il que les caractéristiques de ce MOOC puissent soutenir la motivation des apprenants jusqu'à la fin de la formation. Car si le déploiement de ce dispositif de formation par l'AUF et ses partenaires ne touche que $5 \%$ des participants inscrits, il ne pourra être question de participer de façon importante à la formation des enseignants d'Afrique pour l'usage du numérique en éducation.

\section{La ludification et les MOOC}

Comment améliorer l'engagement des apprenants dans une formation et par la même occasion leur réussite? Nous proposons pour cela d'avoir recours au jeu, qui représente une opportunité éducative forte, notamment par l'intégration d'un aspect ludique aux activités d'apprentissage (Erenli, 2012). C'est ce que nous avons tenté de faire dans le MOOC CERTICE en ayant recours à la ludification (ou gamification en anglais). Ce procédé correspond au transfert de certaines caractéristiques des jeux populaires dans d'autres domaines tels que les activités présentes en formation, y compris en formation à distance (FAD). De nombreux auteurs ont ainsi présenté les stratégies de ludification des apprentissages en FAD (Buckley et Doyle, 2014; Erenli, 2013; Fernandes et al., 2012; Grund, 2015). Enfin, tout en participant au développement de l'autonomie (Bowman, 2015), la ludification peut avoir un impact majeur sur l'un des déterminants de la motivation présentés par Deci et Ryan (2000, voir la section sur la motivation). Dans le cadre des MOOC, la ludification peut impliquer différents changements tels que l'attribution de récompenses pour les ac- tivités d'apprentissage, l'utilisation de niveaux pour susciter la concurrence et l'enjeu, mais aussi la possibilité d'obtenir des badges en participant à des forums, comme le rapportent Morales, AmadoSalvatierra, Hernández, Pirker et Gütl (2016), qui ont étudié les effets de chacune de ces stratégies en contexte MOOC. Leurs travaux montrent une influence positive de la stratégie de récompense dans le dispositif étudié, par rapport aux badges ou au classement par niveaux, les apprenants étant plus motivés à réaliser les activités qui leur étaient assignées. Borras-Gene, Martinez-Nuñez et Fidalgo-Blanco (2016) ont quant à eux montré que le fait d'intégrer des communautés virtuelles et des méthodes de ludification entraînait un développement de la motivation des participants à un MOOC en ingénierie, tout en permettant aux apprenants d'approfondir leurs connaissances et d'augmenter les taux d'achèvement dans le MOOC. Des initiatives ont donc été menées dans le but d'accroître cette réussite des apprenants au MOOC et leur rétention des apprentissages, par exemple celle de Krause, Mogalle, Pohl et Williams (2015) avec le jeu social. Les auteurs ont mis en évidence le fait que les apprenants en situation sociale ont connu une meilleure rétention des informations en regardant les vidéos, mais aussi des performances plus élevées dans leurs scores moyens, par rapport aux étudiants suivant le MOOC en condition normale. Concrètement, dans le MOOC CERTICE, nous utiliserons des capsules vidéo aux dimensions ludiques et réalisées par des enseignants ainsi que des graphiques venant renforcer cet aspect à des fins de ludification du dispositif.

\section{La mobilité et les MOOC}

Pour les apprenants des pays du Sud, la notion de mobilité est devenue une caractéristique essentielle. En effet, l'utilisation du téléphone mobile dans cette région du monde est aujourd'hui largement répandue et on peut donc envisager de parler d'apprentissage nomade, ou de "mobile learning », qui correspond à une nouvelle forme de formation à distance. Celle-ci n'est plus seulement cantonnée à une leçon derrière un ordinateur, mais s'étend au 
téléphone et à la tablette tactile. Et parler de mobilité revient à considérer le dispositif dans son ensemble. En effet, comme le confirment El-Hussein et Cronje (2010), l'apprentissage nomade est à envisager selon trois axes : la mobilité de la technologie; la mobilité de l'apprenant; la mobilité et le dynamisme du processus d'apprentissage. C'est pour cette raison, et pour répondre à la problématique de la forte mobilité des apprenants dans les pays du Sud, que nous nous sommes appuyés sur l'apprentissage nomade dans notre projet, notamment en développant les relations sociales des apprenants et en adaptant l'outil utilisé et le contenu d'apprentissage. En effet, les MOOC peuvent être des lieux d'interaction entre des milliers d'apprenants (Yeager, Hurley-Dasgupta et Bliss, 2013) grâce aux forums et à la participation à une communauté d'apprentissage (certains MOOC ont attiré plus de 10000 étudiants). Mais si l'apprentissage nomade est très apprécié, c'est aussi parce qu'il contient des modules d'apprentissage relativement courts. Ces petites unités, qualifiées de «snack learning» ou d'apprentissage «sur le pouce », permettent d'apprendre à la pause du lunch, dans les transports ou encore en salle d'attente... En effet, les appareils mobiles, tels que les téléphones intelligents, sont aujourd'hui en permanence avec leurs propriétaires ou à proximité immédiate, et ce, 24 heures sur 24 (Sharples, Kloos, Dimitriadis, Garlatti et Specht, 2015). Selon les auteurs, cet accès omniprésent des technologies permet aux apprenants de plonger, chaque fois qu'ils en éprouvent l'intérêt et qu'ils en ont le temps, dans leurs cours. On se rapproche même de plus en plus des applications ludo-éducatives pour téléphones portables proposées dans les boutiques en ligne d'Apple ou de Google. Furió, Juan, Seguí et Vivó (2015) ont ainsi effectué une comparaison entre l'efficacité d'apprentissage et la satisfaction d'apprenants utilisant un jeu sous le format de l'apprentissage nomade avec un iPhone et dans un cours traditionnel. Ils en ont conclu que les deux méthodes utilisées permettaient des gains d'apprentissage significatifs par rapport au cours étudié (le cycle de l'eau) et que le jeu amenait davantage de connaissances aux élèves, mais de manière non significative. Cependant, ils montrent aussi que les élèves ont jugé l'utilisation de l'iPhone bien plus satisfaisante que la leçon en classe. Ainsi, intégrer l'apprentissage nomade n'aura pas uniquement un effet sur le sentiment d'affiliation et de coopération des apprenants, il aura aussi et très certainement un fort impact sur leur motivation.

\section{Les MOOC et le problème de motivation des apprenants}

Pour s'engager dans un MOOC, il s'agit d'être motivé. Et cette motivation serait liée à la performance dans la formation. En effet, comme le rappellent De Barba, Kennedy et Ainley (2016), la motivation, la participation et la performance sont liées dans les cours en ligne ouverts à tous et massifs. Bien qu'il existe plusieurs théories de la motivation, dans cette enquête, c'est la théorie de l'autodétermination (Deci et Ryan, 1985; Ryan et Deci, 2000) qui permettra de poser un regard sur ce concept polysémique (Pintrich et Schunk, 2002). Cette théorie postule que tout individu a une tendance innée à se développer et à s'actualiser, c'est-à-dire qu'il est naturellement porté à agir sur son environnement afin d'accroître ses compétences et son contrôle. La motivation revêt donc une place fondamentale dans cette théorie puisqu'elle permet l'organisation de comportements qui tendent vers l'actualisation du potentiel de chacun. Cette théorie propose deux types de motivation. Celles dites autodéterminées, lorsqu'un individu perçoit qu'il est à l'origine de ses décisions et de ses comportements (Deci et Ryan, 2000), et celles dites contrôlées, lorsqu'un individu perçoit que son comportement lui est imposé par des sources de contrôle interne ou externe. Deci et Ryan (2000) ont théorisé différents types de motivation qui se distinguent par leur degré d'autodétermination, à savoir le degré hypothétique de liberté perçue par l'individu dans le choix et l'exécution de ses actions. Situés sur un continuum d'autodétermination (voir figure 2), ceux-ci sont répartis en trois grands groupes : l'amotivation, la motivation extrinsèque et la motivation intrinsèque. 


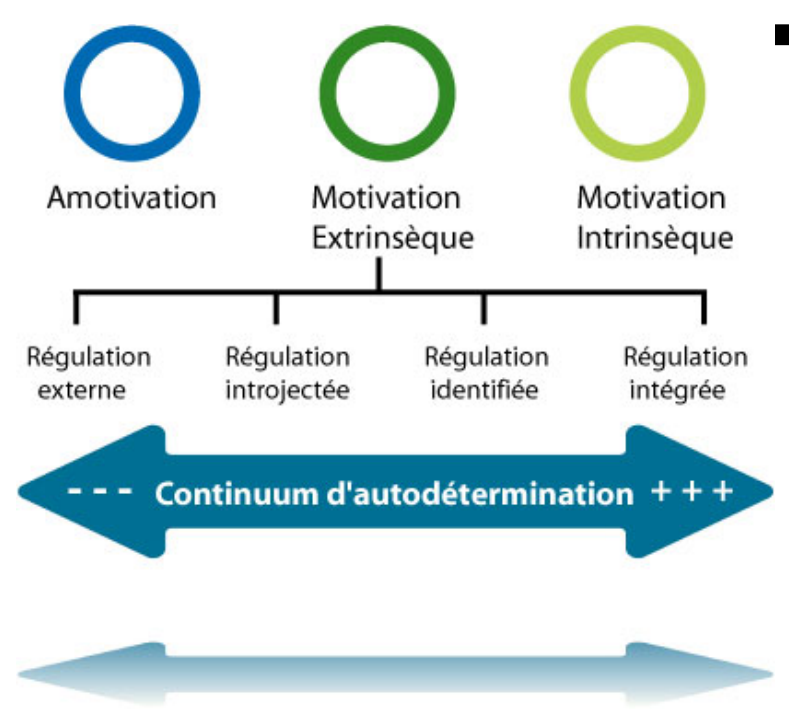

Figure 2: Schématisation de la théorie de l'autodétermination de Deci et Ryan (2000)

Pour Deci et Ryan (2000), les diverses formes de la motivation intrinsèque font partie des motivations dites autodéterminées. Par ailleurs, la motivation autodéterminée est associée à plus d'efficacité (Deci et Ryan, 2000), notamment sur le plan scolaire (Guay, Ratelle, Roy et Litalien, 2010). Cela trouve un écho particulier dans ce MOOC concernant des enseignants ou de futurs enseignants. En effet, il a été prouvé que la motivation jouait un rôle important pour ce public en matière de formation continue et de développement professionnel (Schieb et Karabenick, 2011). De plus, la grande importance de l'aspect social dans la motivation des apprenants a été mise en évidence. En effet, le fait qu'il y ait des interactions entre pairs réduirait le décrochage des apprenants, comme le confirment Barak, Watted et Haick (2016). On comprend bien alors l'importance de mettre en place des stratégies permettant de développer la motivation des apprenants engagés dans un MOOC, et ce, notamment, en stimulant leur investissement dans le cours, en recourant par exemple à la ludification des contenus ou encore à l'apprentissage nomade.

\section{Méthodologie}

En accord avec les objectifs de cette recherche, soit 1) décrire le rôle de la ludification des apprentissages et de la mobilité (apprentissage nomade) dans la motivation des participants à un MOOC, et 2) établir d'autres caractéristiques d'un MOOC susceptibles de participer à la motivation des apprenants, cette section présente la méthodologie de la recherche. Comme le préconise la $6^{\mathrm{e}}$ édition du Publication Manual of the American Psychological Association (2013), on y retrouve la présentation des participants, de l'instrument de collecte de données et des stratégies d'analyse des données recueillies. Une section sur les forces et les limites méthodologiques de cette recherche vient clore cette partie méthodologique.

\section{Participants}

Cette recherche a analysé des données provenant de deux cohortes d'apprenants d'Afrique qui ont participé au MOOC CERTICE. Les données ont été recueillies auprès de la première cohorte $(n=2131)$ en septembre 2015 et auprès de la deuxième cohorte $(n=2538)$ en septembre 2016. Comme les caractéristiques démographiques des participants de la cohorte 1 étaient similaires à celles des participants de la cohorte 2, nous les présentons de façon groupée. L'âge moyen des participants était de 29,2 ans; $11,5 \%$ étaient des femmes et $88,5 \%$ des hommes. Le tableau 1 illustre le fait que les participants provenaient de 26 pays différents d'Afriques.

$5 \quad$ Seuls les participants ayant indiqué avoir un pays de résidence en Afrique ont été pris en considération dans l'enquête. 
Tableau 1 : Principales caractéristiques des participants

\begin{tabular}{|l|l|}
\hline Pays & Nombre de participants \\
\hline Algérie & 1374 \\
\hline Bénin & 83 \\
\hline Burkina Faso & 78 \\
\hline Burundi & 6 \\
\hline Cameroun & 82 \\
\hline Congo & 23 \\
\hline Côte d'Ivoire & 321 \\
\hline Égypte & 2 \\
\hline Éthiopie & 1 \\
\hline Gabon & 33 \\
\hline Ghana & 2 \\
\hline Guinée & 21 \\
\hline Kenya & 1 \\
\hline Madagascar & 9 \\
\hline Mali & 82 \\
\hline Maroc & 1343 \\
\hline Mauritanie & 6 \\
\hline Niger & 37 \\
\hline République centrafricaine & 2 \\
\hline République démocratique du \\
Congo & 127 \\
\hline Rwanda & 4 \\
\hline Sénégal & 232 \\
\hline Soudan & 1 \\
\hline Tchad & 5 \\
\hline Togo & 53 \\
\hline Tunisie & 741 \\
\hline
\end{tabular}

La différence entre le nombre de participants en Afrique du Nord (Maghreb) et en Afrique subsaharienne peut s'expliquer notamment par le nombre d'étudiants inscrits à l'université dans les pays du Maghreb mais aussi par le degré de connectivité. En effet, il y a une proportion bien plus grande d'étudiants universitaires en Afrique du Nord qu'en Afrique subsaharienne. À titre d'exemple, il y avait 1,5 million d'étudiants à l'université en Algérie en 2015-2016, pour une population de 39,5 millions d'habitants (soit $4 \%$ de la population inscrite à l'université) ${ }^{6}$. En Afrique, de telles statistiques sont pratiquement impossibles à atteindre. Au Burkina Faso, c'est ainsi $0,4 \%$ de la population qui est ins-

\footnotetext{
6 http://www.aps.dz/sante-sciences-tech/28096rentr $\% \mathrm{C} 3 \% \mathrm{~A} 9 \mathrm{e}-$ universitaire-pr $\% \mathrm{C} 3 \% \mathrm{~A} 8 \mathrm{~s}$-de-1,5-million-d-\% $\%$ C $\%$ A 9 tudiants-rejoindront-dimanche-leurs\%C3\%A9tablissements
}

crite à l'université7, soit 10 fois moins d'étudiants universitaires qu'en Algérie. Par ailleurs, sur le plan de la connectivité, on peut voir que les pays du Maghreb bénéficient de bien meilleures infrastructures que ceux du Sud. Si l'on se réfère à l'Indice de développement des TIC (IDI) pour 2012 et $2013^{8}$, on constate, pour rester sur nos exemples précédents, que l'Algérie était, en 2015, au 114e rang alors que le Burkina Faso était au $156^{\mathrm{e}}$ rang. Enfin, il se peut que d'autres facteurs aient également influencé cette tendance, par exemple la présence au Maghreb de l'IFIC $^{9}$ qui a largement fait la promotion du MOOC CERTICE.

La figure 3 présente le plus haut diplôme obtenu par les participants au MOOC CERTICE. Les données recueillies dans le cadre de cette enquête confirment celles d'autres enquêtes qui ont révélé que les participants à un MOOC ont généralement tous déjà un diplôme universitaire (Karsenti, 2013). En effet, ce sont ici 95,4\% des participants inscrits au MOOC CERTICE qui possèdent déjà un diplôme universitaire de $1^{\text {er }}$ cycle.

\footnotetext{
7 http://www.cns.bf/IMG/pdf/tableau_de bord_superieur $\underline{\text { 2012 2013.pdf }}$

8 http://www.itu.int/net/pressoffice/press_releases/2014/68$\underline{\text { fr.aspx }}$

9 http://ific.auf.org (IFIC = Institut de la Francophonie pour l'ingénierie de la connaissance et la formation à distance)
} 


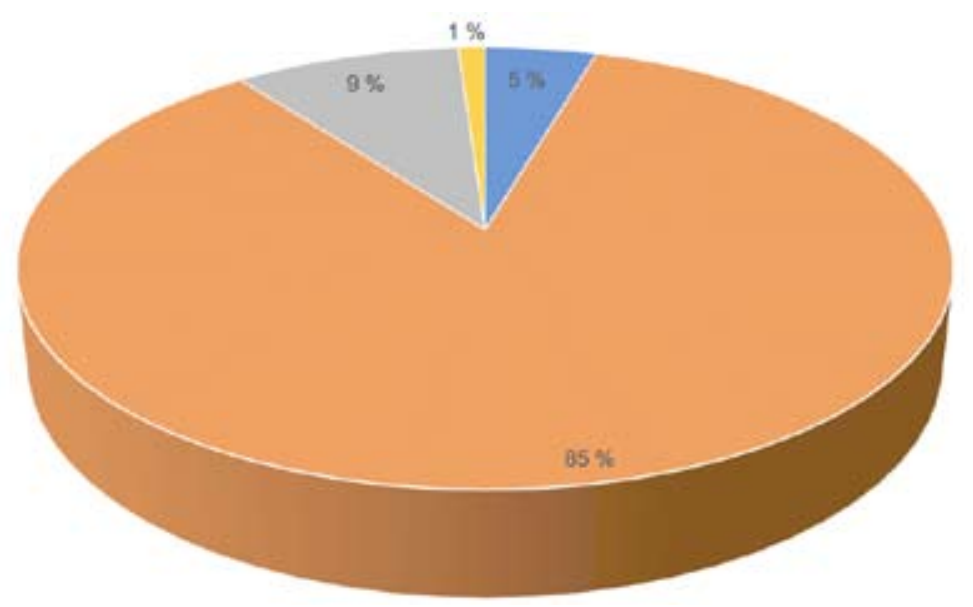

- Secondaire

- Prarrier cycla universitaira

- Deuxieme sycle universitaire

- Troisiême cycle untversiltaire

Figure 3 : Plus haut diplôme obtenu avant l'inscription au MOOC CERTICE

La figure 4 présente la principale occupation des apprenants inscrits au MOOC. On constate ainsi que $54 \%$ des participants sont enseignants, $35 \%$ sont étudiants, et $11 \%$ ont une autre occupation principale.

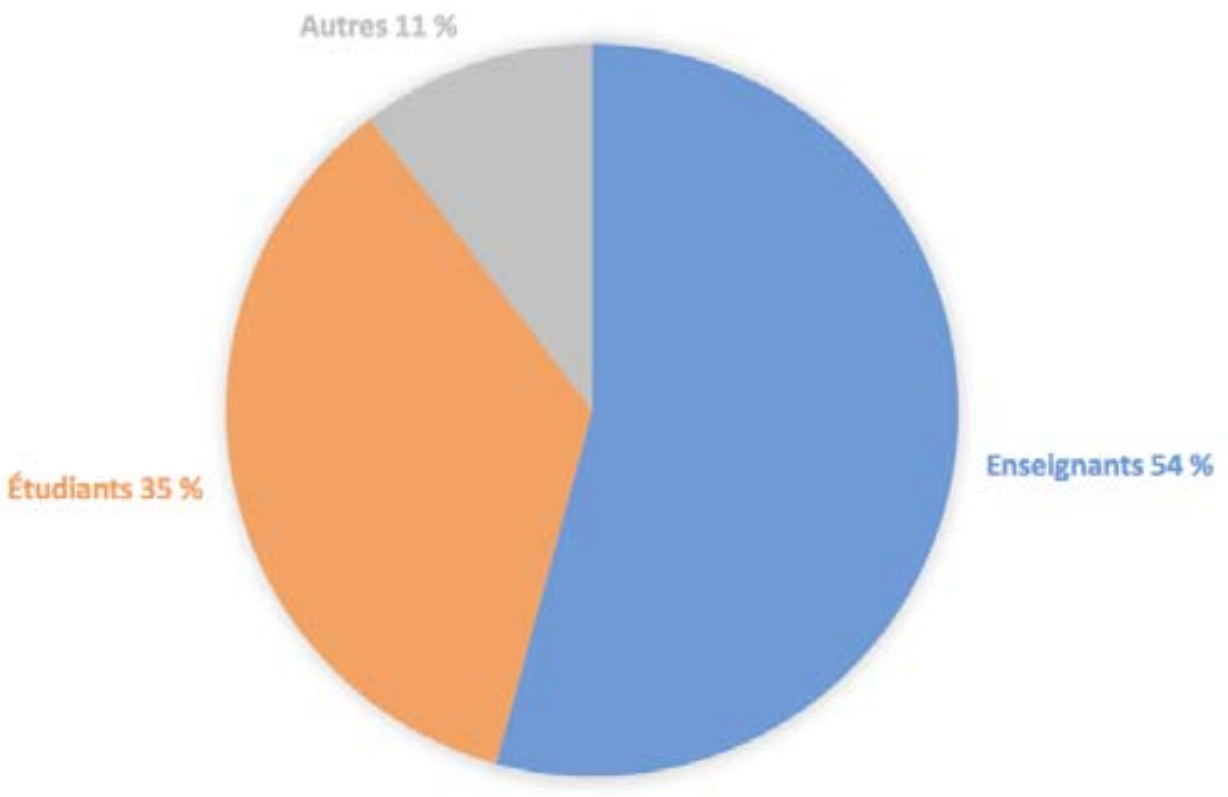

Figure 4: Principale occupation des apprenants inscrits au

MOOC CERTICE 


\section{Outil de collecte de données}

Un questionnaire a été développé à partir des indicateurs présentés par Ho et al. (2014) de même que Kerr, Houston et Marks (2015), relativement à l'impact potentiel du MOOC sur la motivation des apprenants en fonction notamment des trois déterminants établis par Deci et Ryan (2000). Le questionnaire utilisé ${ }^{10}$ portait notamment sur :

- L'impact global du MOOC sur la motivation;

- L'impact du MOOC sur le sentiment de compétence des apprenants;

- L'impact de la ludification des apprentissages sur la motivation des apprenants;

- L'impact de l'apprentissage nomade sur la motivation des apprenants;

- L'impact des caractéristiques sociales du MOOC sur la motivation des apprenants.

Ce questionnaire comportait également des questions plus spécifiques sur la thématique du MOOC CERTICE, par exemple la perception des participants de l'impact du MOOC sur leur usage du numérique dans leur pratique d'enseignement. Ces aspects de l'enquête seront abordés dans une publication future. Pour chacune des cohortes, un premier message a été envoyé de même que deux rappels afin qu'elles participent à l'enquête. Par ailleurs, le questionnaire est resté accessible en ligne pendant une période totale de 15 jours pour chacune des deux cohortes.

\section{Analyse des données}

Les données recueillies à l'aide du questionnaire comprennent à la fois des échelles de Likert et des réponses à des questions semi-ouvertes ou ouvertes. Par conséquent, l'analyse qui en ressort est dite de type mixte. L'analyse quantitative comprend des statistiques descriptives élaborées à l'aide du logiciel SPSS $23^{11}$ et de l'outil de sondage en ligne Sur-

\footnotetext{
10 Cette enquête comportait également d'autres questions posées aux participants. Elles seront abordées dans une publication ultérieure.

11 http://www-01.ibm.com/software/analytics/spss/
}

veyMonkey ${ }^{12}$. Ces premiers résultats d'analyse sont approfondis et appuyés par une analyse qualitative des réponses ouvertes aux questionnaires, effectuée à l'aide du logiciel $Q D A$ Miner $^{13}$. Celle-ci consiste en une analyse de contenu (L'Écuyer, 1990; Miles et Huberman, 2003) dont le codage semi-ouvert a été construit à partir des réponses des participants en fonction des deux objectifs de recherche.

\section{Forces et limites méthodologiques}

L'une des principales forces de la présente étude réside assurément dans la méthodologie de recherche particulière utilisée : l'enquête en ligne qui a permis de rejoindre un nombre important de participants dans quelque 26 pays d'Afrique. Ce choix méthodologique n'est pourtant pas sans limites. Tout d'abord, le fait de travailler à partir des perceptions constitue une limite importante que nous avons tenté de pallier par un vaste échantillon de participants et des méthodes d'analyse de données variées. De plus, pour réduire ce biais méthodologique, les analyses effectuées ont systématiquement comparé les réponses des différents types de répondants, mettant en exergue leurs points de divergence lorsque nécessaire. Une autre limite de l'étude est liée à l'échantillon des participants, qui n'était pas aléatoire, c'est-à-dire que notre choix des participants n'avait pas pour objectif de représenter un sous-ensemble de la population interrogée (les apprenants d'Afrique qui participaient à un MOOC). Pourquoi? Parce que dans notre contexte, il semblait particulièrement difficile, voire impossible de procéder à une sélection aléatoire des participants. En effet, comment aurions-nous pu obliger des participants au MOOC à prendre part à l'enquête? Nous avons plutôt misé sur un échantillon de participants volontaires, soit un échantillon non probabiliste qui n'aspire pas à être représentatif, mais simplement à utiliser les répondants disponibles ou volontaires et aisément interrogeables. La seule restriction en ce qui a trait aux participants était d'avoir suivi le MOOC CERTICE.

$\begin{array}{ll}12 & \underline{\text { https://www.surveymonkey.com/ }} \\ 13 & \text { http://provalisresearch.com/fr/produits/logiciel-d- } \\ & \text { analyse-qualitative/ }\end{array}$




\section{Résultats}

Les données recueillies auprès de 4669 participants de deux cohortes d'apprenants au MOOC CERTICE révèlent que $83,9 \%$ d'entre eux perçoivent que les caractéristiques de la formation ont eu un impact globalement positif sur leur motivation (figure 5).

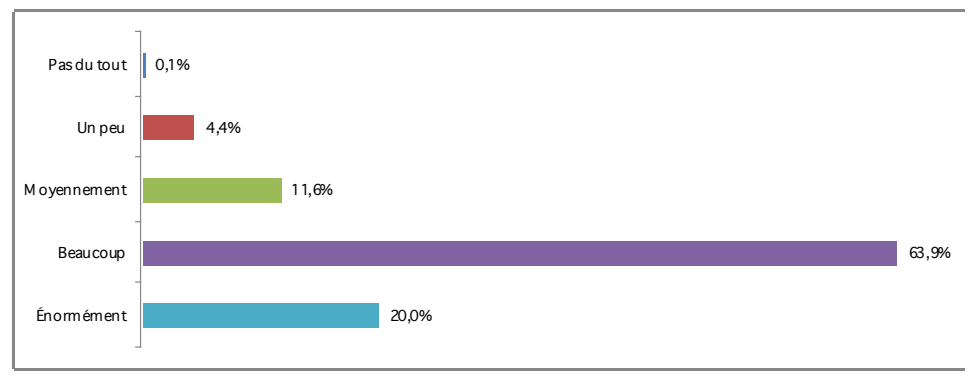

Figure 5: Impact global des caractéristiques du M00C sur la motivation des apprenants

Les données recueillies par l'entremise du questionnaire d'enquête en ligne révèlent également un impact relativement modeste des caractéristiques du MOOC sur le sentiment de contrôle des apprenants (figure 6). En effet, seuls $25,7 \%$ des participants indiquent que le MOOC a eu «beaucoup » ou " énormément» d'impact sur leur sentiment de compétence. Cela s'explique peut-être par la nature même de l'activité où les participants, même s'ils avaient certains choix, étaient néanmoins contraints de suivre un nombre fixe de modules. Cela dit, et malgré ce contexte, les résultats révèlent tout de même que seuls $11,4 \%$ des participants ne perçoivent pas que le MOOC participe d'une manière quelconque à leur sentiment de contrôle.

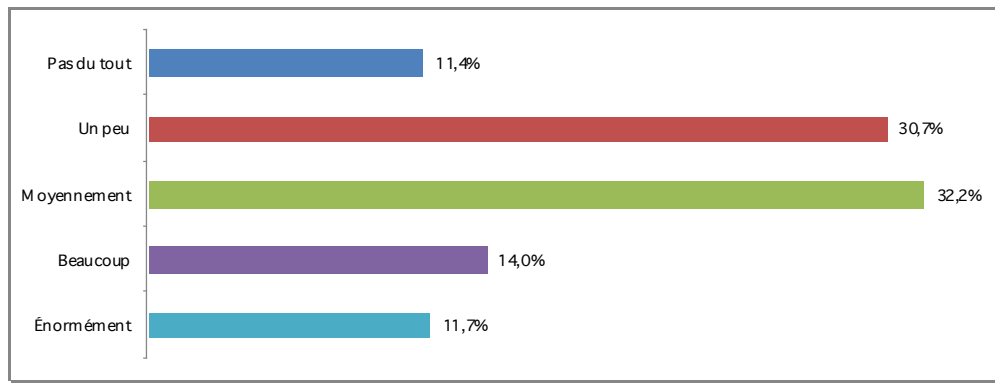

Figure 6 : Impact des caractéristiques du M00C sur le sentiment de contrôle des apprenants
Les données recueillies ont également permis de voir que les caractéristiques du MOOC avaient un fort impact sur le sentiment de compétence des participants. En effet, ils sont 98,2\% à affirmer que le MOOC a eu «beaucoup » ou " énormément» d'impact sur leur sentiment de compétence, relativement à la thématique du cours, soit l'usage du numérique en éducation (figure 7). Ce résultat est particulièrement intéressant puisqu'il montre que l'un des principaux déterminants de la motivation de Deci et Ryan (1985), le sentiment de compétence, semble grandement favorisé par la participation au MOOC. En revanche, il est possiblement normal de concevoir qu'un tel cours, dans un domaine encore peu connu des participants (le numérique en éducation), puisse avoir un tel impact. Si le MOOC portait sur une autre thématique, les résultats auraient peut-être été fort différents.

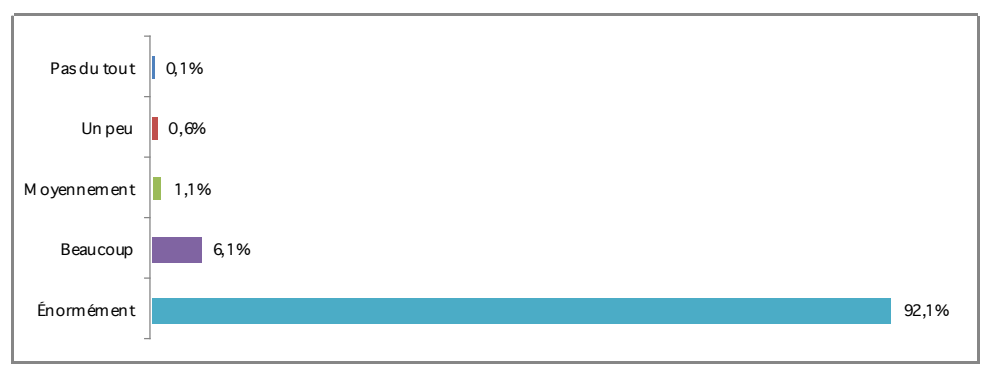

Figure 7: Impact global des caractéristiques du M00C sur le sentiment de compétence des apprenants

Plusieurs caractéristiques « sociales » étaient intégrées au MOOC CERTICE : moments d'échanges et de collaboration entre les participants, rencontres en présentiel des apprenants dans des campus numériques de l'Agence universitaire de la Francophonie, séances de LiveTweet, etc. Ces éléments avaient notamment pour but de favoriser le sentiment d'affiliation des apprenants. Les données recueillies ont permis de comprendre le rôle que ces caractéristiques sociales du MOOC ont eu sur la motivation des apprenants (figure 8). En effet, ce sont $70,3 \%$ des participants qui indiquent qu'elles ont eu « beaucoup » ou " énormément » d'impact sur leur motivation. 


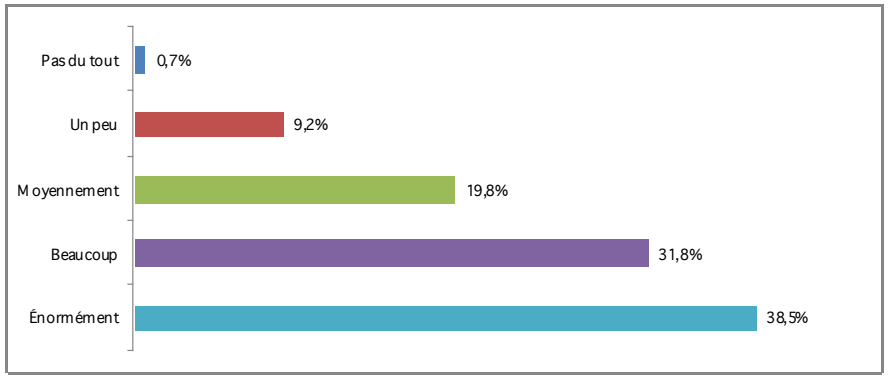

Figure 8 : Impact des caractéristiques sociales du M00C sur la motivation des apprenants

Les réponses des quelque 4669 participants ont permis de mieux comprendre les divers avantages des caractéristiques sociales présentes dans le MOOC (figure 9). Une analyse qualitative avec le logiciel QDA Miner révèle par exemple en quoi la présence de caractéristiques sociales comporte de nombreux avantages pour les apprenants. Cette analyse qualitative permet, entre autres, de voir le rôle des caractéristiques sociales dans la motivation des apprenants $(28,3 \%$ des participants ont souligné que les caractéristiques sociales participent à leur motivation). Les participants ont aussi indiqué que les caractéristiques sociales du MOOC leur permettaient de briser l'isolement $(31,8 \%)$, de trouver plus facilement de l'aide $(29,5 \%)$, de réaliser des travaux d'équipe $(7,2 \%)$, voire de se situer par rapport aux autres participants $(6,9 \%)$.

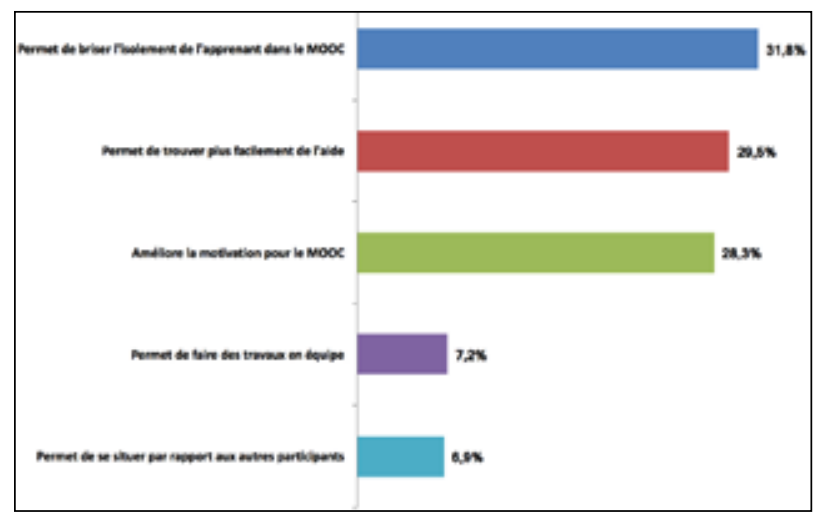

Les caractéristiques liées à la ludification des apprentissages présentes dans le MOOC (notamment les images, les dessins, les vidéos, de même que certaines activités) semblent aussi avoir participé à la motivation des apprenants (figure 10). En effet, ils sont $87,5 \%$ à avoir indiqué que la ludification des apprentissages a eu « beaucoup » ou « énormément » d'impact sur leur motivation.

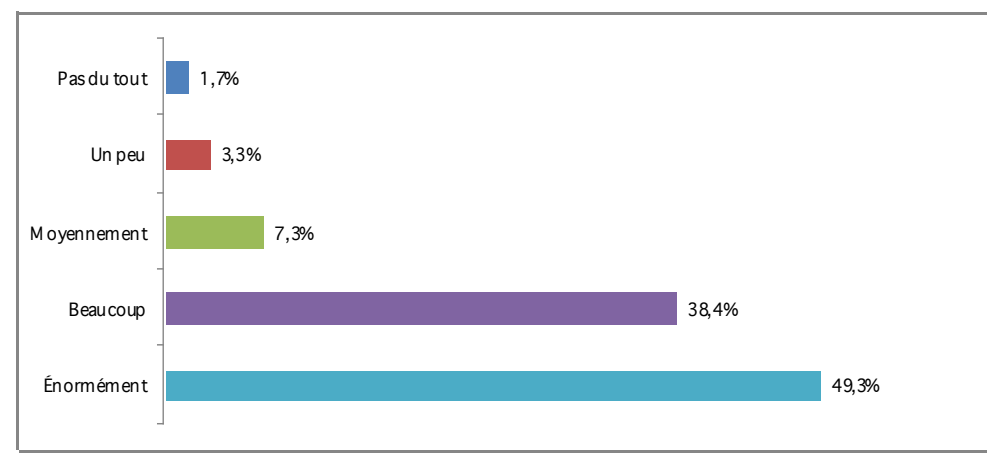

Figure 10: Impact des éléments de ludification du M00C sur la motivation des apprenants

Figure 9 : Principaux avantages liés aux caractéristiques sociales du MOOC, selon les participants ${ }^{14}$

14 Note méthodologique : Cette figure présente l'analyse qualitative (de contenu) réalisée à l'aide du logiciel QDA Miner. Les répondants avaient la possibilité de ne nommer qu'un seul ou plusieurs avantages liés aux différentes caractéristiques du MOOC. L'analyse a permis de faire ressortir les principaux avantages, présentés dans les figures. Les pourcentages ne doivent en aucun cas être additionnés pour totaliser $100 \%$. Ils représentent plutôt l'importance relative accordée à chacune des raisons par les participants. 
Les données recueillies ont également permis de mieux comprendre les divers avantages de cette ludification faisant partie du MOOC CERTICE (figure 11). En effet, une analyse qualitative avec le logiciel QDA Miner révèle en quoi la ludification des apprentissages revêt un caractère positif pour les apprenants. Cette analyse permet notamment de confirmer le rôle de la ludification dans la motivation des apprenants, puisqu'il s'agit du premier avantage invoqué (61,4\% des participants). Les participants ont aussi indiqué que la ludification rendait la participation au MOOC plus agréable $(53,4 \%)$, que cela leur permettait de mieux comprendre les concepts présentés $(32,8 \%)$, ou encore que cela leur permettait d'apprendre d'une façon différente $(15,7 \%)$.

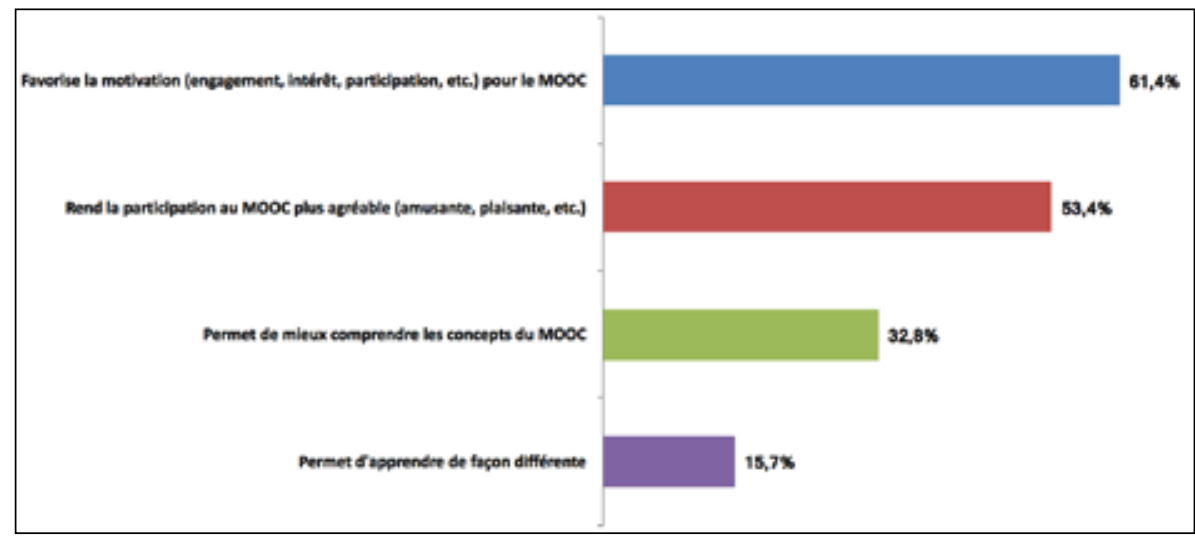

Figure 11: Principaux avantages liés à la ludification des apprentissages selon les participants

Les caractéristiques de l'apprentissage nomade (messages textes d'encouragements, messages textes de rappels, messages textes de bilans, messages textes de résultats) semblent non seulement très appréciées des participants, mais elles sont aussi susceptibles d'avoir un impact sur leur motivation pour le MOOC. En effet, ce sont 90,1\% des participants qui indiquent que l'apprentissage nomade a « beaucoup » ou « énormément » d'impact sur leur motivation (figure 12).

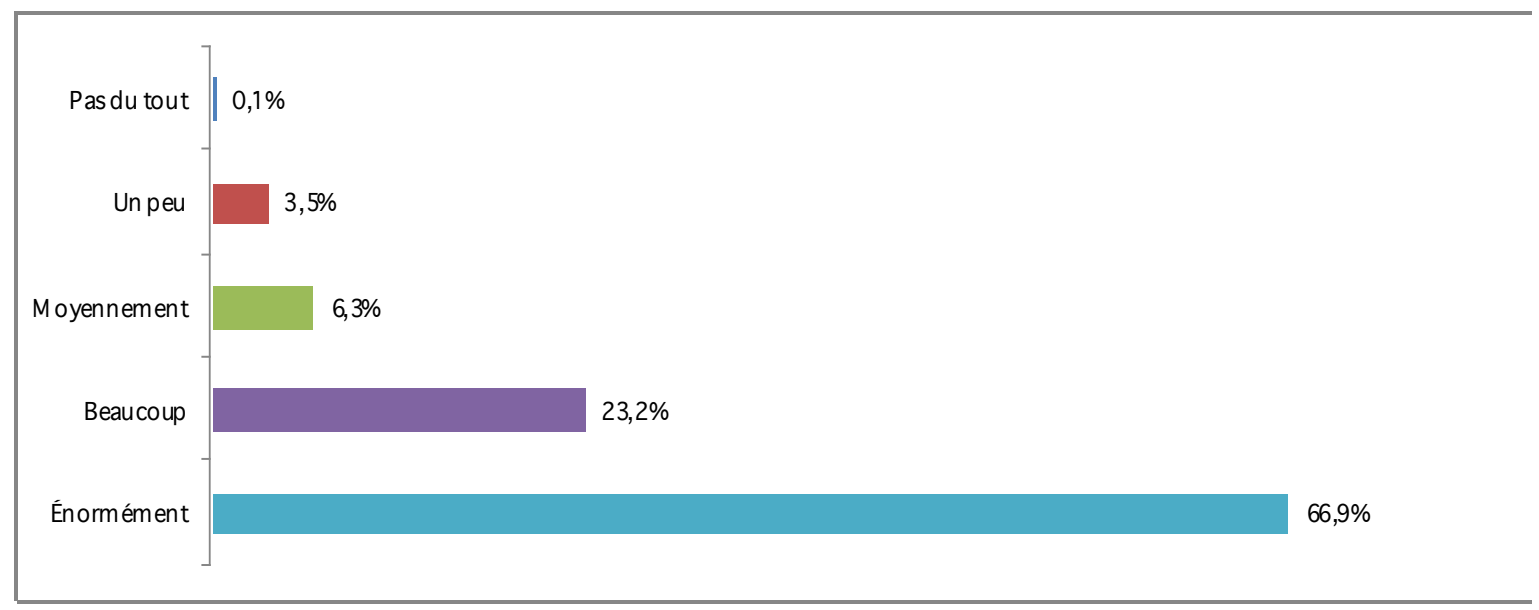

Figure 12: Impact global des éléments d'apprentissage nomade du M00C sur la motivation des apprenants 
Comme l'apprentissage nomade dans le cadre du MOOC CERTICE se résumait principalement à l'envoi de messages textes, il a été demandé aux participants de comparer ce moyen de communication aux autres mis parallèlement en place (courriel, forum, foire aux questions, etc.). Sur ce point, les réponses des participants sont particulièrement révélatrices de leur appréciation de ce moyen de communication, en ce qui concerne leur motivation pour le MOOC. En effet, comme le montre la figure 13, ce sont 98,2 \% des participants qui disent préférer « beaucoup » ou « énormément » communiquer par message texte dans le cadre du MOOC plutôt que par un autre moyen de communication.

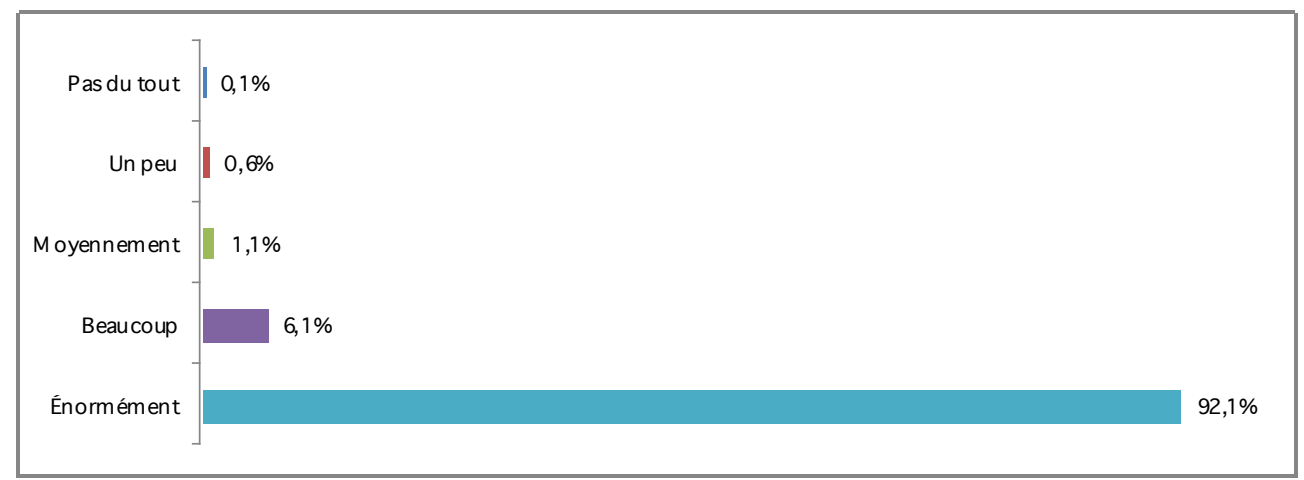

Figure 13: Comparaison de l'appréciation de la communication par message texte par rapport à d'autres moyens (courriel, forum, FAQ) dans le cadre du MOOC

Une analyse qualitative (avec le logiciel $Q D A$ Miner) des données issues du questionnaire auprès de deux cohortes de participants au MOOC CERTICE a permis de mieux comprendre les nombreux avantages que les apprenants perçoivent dans l'apprentissage nomade (figure 14). Ces résultats confirment d'abord l'impact de l'apprentissage nomade sur la motivation des apprenants pour un MOOC, puisqu'il s'agit d'un avantage souligné par 40,7\% des répondants. Le fait de gagner du temps arrive toutefois en tête des raisons invoquées par les participants (49,5\%). L'idée de rester en «contact avec le MOOC en tout temps » $(43,5 \%)$ ou encore la facilitation de la participation (39,3\%) font également partie des principaux avantages attribués à l'apprentissage nomade $(43,5 \%)$. De nombreux participants $(28,7 \%)$ ont indiqué que ce type d'apprentissage éliminait, en partie, les problèmes de connectivité à Internet, qui sont encore très fréquents en Afrique. Ainsi, selon eux, le fait de pouvoir communiquer par message texte les rend plus autonomes dans leur apprentissage. Rappelons ici que le sentiment d'autonomie ou d'autodétermination fait partie des trois déterminants de la motivation selon Deci et Ryan $(1985,2000)$.

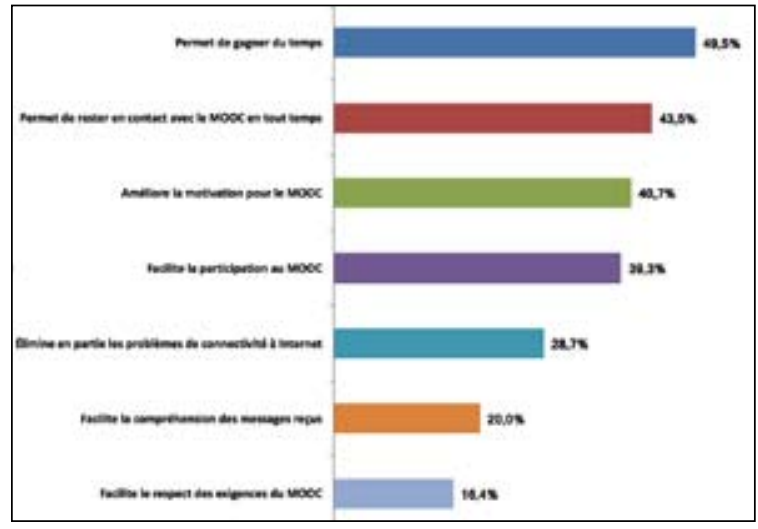

Figure 14: Principaux avantages liés à l'apprentissage nomade selon les participants 
Parmi les autres principaux avantages indiqués par les participants, on retrouve une meilleure compréhension des messages reçus $(20,0 \%)$, de même qu'un meilleur respect des exigences du MOOC $(16,4 \%)$. Ce dernier avantage est irrévocablement lié aux messages textes de rappels reçus par les participants.

\section{Conclusion}

Les résultats de l'enquête réalisée auprès de deux cohortes d'apprenants du MOOC CERTICE révèlent que les caractéristiques de cette formation en ligne participent de façon générale à la motivation des apprenants. Ces derniers se sentaient notamment plus autodéterminés et plus compétents dans ce cours, deux déterminants majeurs de la motivation pour Deci et Ryan (2000). Les résultats montrent également que les caractéristiques sociales du MOOC semblent être grandement appréciées des apprenants, en plus de jouer un rôle certain dans leur sentiment d'appartenance de ces derniers. Il s'agit là du troisième déterminant de la motivation de Deci et Ryan (2000). La ludification semble également jouer un rôle dans leur motivation. En effet, lorsqu'on leur demande quels sont les principaux avantages de la ludification (voir figure 11), ils indiquent que cela rend l'apprentissage plus motivant et plus agréable. La ludification leur permet aussi de mieux comprendre - un lien certain avec leur sentiment de compétence -, voire d'apprendre différemment. Ils sont même $87,7 \%$ à estimer que la ludification a beaucoup, voire énormément d'influence sur leurs apprentissages. Ces éléments confirment la forte influence de la ludification sur la motivation des apprenants.

Mais ce sont véritablement les caractéristiques inhérentes à l'apprentissage nomade - notamment par l'usage de messages textes d'encouragements, de messages textes de rappels, de messages textes de bilans et de messages textes de résultats - qui semblent avoir été le plus appréciées parmi les 4669 apprenants interrogés. En effet, ce sont plus de $90 \%$ d'entre eux qui soulignent que cette forme de mobilité participe «beaucoup » ou " énormément » à leur motivation pour le MOOC.
Ces avantages de la mobilité s'expliquent d'ailleurs de diverses façons pour les apprenants : cela leur permet de gagner du temps, de rester en contact avec le cours en tout temps, d'être plus motivés, de participer plus facilement, d'atténuer les problèmes de connectivité à Internet, voire de mieux comprendre les messages (courts) reçus ou encore de mieux respecter les exigences relatives aux travaux.

Ces résultats positifs pourraient toutefois s'expliquer par plusieurs raisons. Premièrement, le MOOC CERTICE de l'AUF est l'un des tout premiers MOOC spécialement conçus pour les apprenants africains. Ce MOOC a été en grande partie conçu par des experts d'Afrique, et les exemples présentés proviennent tous de ce continent, contrairement à d'autres MOOC où la plupart des exemples présentés proviennent des États dits du Nord. Cela pourrait, déjà, expliquer en partie ces résultats positifs.

Deuxièmement, il faut comprendre que le MOOC CERTICE venait répondre à un besoin pressant en Afrique francophone. En effet, la formation dans le domaine du numérique en éducation est particulièrement recherchée par les enseignants africains, et comme très peu d'universités d'Afrique offrent de telles formations, l'engouement pour le MOOC CERTICE peut aussi être mieux compris.

Troisièmement, la thématique même du MOOC CERTICE - le numérique - est particulièrement populaire dans notre société de l'information. Il s'agit d'ailleurs de la thématique la plus populaire dans tous les MOOC proposés par Coursera, edX ou Udacity (Karsenti, 2015). Nous vivons à une époque de mutations rapides où les apprenants d'Afrique sont captivés par le numérique, qui influence l'évolution de l'ensemble des sociétés et affecte de façon significative toutes les dimensions économiques, sociales, culturelles ou éducatives.

Lors de recherches futures, il pourrait être intéressant de réaliser des études quantitatives, avec des échelles de motivation fondées sur le modèle de Deci et Ryan (2000), afin d'avoir un autre regard sur l'impact des caractéristiques des MOOC sur la motivation des apprenants. 
Enfin, il semble important de souligner, pour conclure, que le MOOC CERTICE de l'AUF est susceptible de jouer un rôle majeur dans l'éducation en Afrique. En effet, c'est par cette formation proposée gratuitement à tous les enseignants (et autres) francophones d'Afrique qu'il sera peut-être possible d'envisager un virage numérique dans les écoles de ce continent. Car, faut-il le rappeler, pour l'OCDE (2015), le numérique représente « l'avenir même » de l'éducation. En outre, étant donné l'omniprésence sociale des technologies, leur maîtrise par les nouvelles générations semble de plus en plus déterminante pour assurer leur réussite sociale et professionnelle. En effet, il y a tout lieu de croire que le fait de savoir s'autoformer, s'informer et communiquer à l'aide de différentes technologies est désormais une condition essentielle pour pouvoir s'adapter à une société en mutation constante et devenir des acteurs sociaux à part entière. L'usage des technologies représente donc actuellement une compétence-clé pour permettre aux jeunes et moins jeunes de mieux réussir en contexte éducatif et, plus largement, dans la société du savoir dans laquelle nous vivons. Et il semble que le MOOC CERTICE de l'AUF participe résolument à cela.

\section{Références}

Achard, P. (2016). Les MOOCs : cours en ligne et transformations des universités. Montréal, Canada : Presses de l'Université de Montréal.

Acosta, E. S., Otero, J. J. E. et Valderrama, F. (2014). Motivation in massive education online development and testing of a system of accreditation badges for MOOC. Digital Education Review, (25), 18-35. Récupéré de http://revistes.ub.edu

Alraimi, K. M., Zo, H. et Ciganek, A. P. (2015). Understanding the MOOCs continuance: The role of openness and reputation. Computers \& Education, 80, 28-38. https://doi.org/10.1016/j.compedu.2014.08.006

Barak, M., Watted, A. et Haick, H. (2016). Motivation to learn in massive open online courses: Examining aspects of language and social engagement. Computers \& Education, 94, 49-60. https://doi.org/10.1016/j.compedu.2015.11.010
Borras-Gene, O., Martinez-Nuñez, M. et Fidalgo-Blanco, A. (2016). New challenges for the motivation and learning in engineering education using gamification in MOOC. International Journal of Engineering Education, 32(1), 501512. Récupéré du site du Research Group in InterAction and eLearning of the University of Salamanca : http://repositorio.grial.eu

Bowman, N. K. (2015, mars). Investigating the impact of a gamified unit of instruction on student motivation and learning. Communication présentée à la $20^{\text {th }}$ Annual Technology, Colleges, and Community Worldwide Online Conference. Récupéré de l'archive ScholarSpace : http://scholarspace.manoa.hawaii.edu

Breslow, L., Pritchard, D. E., DeBoer, J., Stump, G. S., Ho, A. D. et Seaton, D. T. (2013). Studying learning in the worldwide classroom research into edX's first MOOC. Research \& Practice in Assessment, 8, 13-25. Récupéré de http://www.rpajournal.com

Buckley, P. et Doyle, E. (2014). Gamification and student motivation. Interactive Learning Environments, 24(6), 1162-1175. https://doi.org/10.1080/10494820.2014.964263

Bulger, M., Bright, J. et Cobo, C. (2015). The real component of virtual learning: Motivations for face-to-face MOOC meetings in developing and industrialised countries. Information Communication and Society, 18(10), 1200-1216. https://doi.org/10.1080/ 1369118x.2015.1061571

Chang, R. I., Hung, Y. H. et Lin, C. F. (2015). Survey of learning experiences and influence of learning style preferences on user intentions regarding MOOCs. British Journal of Educational Technology, 46(3), 528-541. https://doi.org/10.1111/bjet.12275

De Barba, P. G., Kennedy, G. E. et Ainley, M. D. (2016). The role of students' motivation and participation in predicting performance in a MOOC. Journal of Computer Assisted Learning, 32(3), 218-231. https://doi.org/10.1111/jcal.12130

Deci, E. L. et Ryan, R. M. (1985). Intrinsic motivation and self-determination in human behavior. New York, NY : Plenum. 
Deci, E. L. et Ryan, R. M. (2000). The "what" and "why" of goal pursuits: Human needs and the self-determination of behavior. Psychological Inquiry, 11(4), 227-268. https://doi.org/10.1207/s15327965pli1104_01

El-Hussein, M. O. M. et Cronje, J. C. (2010). Defining mobile learning in the higher education landscape. Educational Technology \& Society, 13(3), 12-21. Récupéré de http://ifets.info

Erenli, K. (2012). The impact of gamification: A recommendation of scenarios for education. Dans Proceedings of the 15th International Conference on Interactive Collaborative Learning (ICL) (p. 1-8). IEEE. https://doi.org/10.1109/icl.2012.6402106

Erenli, K. (2013). The impact of gamification - Recommending education scenarios. International Journal of Emerging Technologies in Learning (iJET), 8(S1), 15-21. https://doi.org/10.3991/ijet.v8is1.2320

Fernandes, J., Duarte, D., Ribeiro, C., Farinha, C., Pereira, J. M. et da Silva, M. M. (2012). iThink: A game-based approach towards improving collaboration and participation in requirement elicitation. Procedia Computer Science, 15, 66-77. https://doi.org/10.1016/j.procs.2012.10.059

Flückiger, Y. et Achard, P. (2016). From MOOCs to MOORs: A movement towards Humboldt 2.0. Dans L. E. Weber et J. Duderstadt (dir.), University priorities and constraints (p. 301311). Paris, France : Economica. Récupéré de http://archive-ouverte.unige.ch

Freitas, S. I., Morgan, J. et Gibson, D. (2015). Will MOOCs transform learning and teaching in higher education? Engagement and course retention in online learning provision. British Journal of Educational Technology, 46(3), 455-471. https://doi.org/10.1111/bjet.12268

Furió, D., Juan, M. C., Seguí, I. et Vivó, R. (2015). Mobile learning vs. traditional classroom lessons: A comparative study. Journal of Computer Assisted Learning, 31(3), 189-201. https://doi.org/10.1111/jcal.12071

García Espinosa, B. J., Tenorio Sepúlveda, G. C. et Ramirez Montoya, M. S. (2015). Self-motivation challenges for student involvement in the open educational movement with MOOC. RUSC. Universities and Knowledge Society Journal, 12(1), 91-103.
Gillani, N. (2013). Learner communications in massively open online courses (OxCHEPS Occasional Paper No. 53). Récupéré du site de l'Oxford Centre for Higher Education Policy Studies : http://oxcheps.new.ox.ac.uk

Goldberg, L. R., Bell, E., King, C., O’Mara, C., McInerney, F., Robinson, A. et Vickers, J. (2015). Relationship between participants' level of education and engagement in their completion of the Understanding Dementia Massive Open Online Course approaches to teaching and learning. BMC Medical Education, 15(1),1642. https://doi.org/10.1186/s12909-015-0344-z

Gray, C. (2015). Exploring measures of engagement in open online work based learning: Towards better measures of success in open online learning methods, massive or otherwise. International Journal of Technologies in Learning, 22(3), 41-50. https://doi.org/10.18848/23270144/cgp/v22i03/49169

Greene, J. A., Oswald, C. A. et Pomerantz, J. (2015). Predictors of retention and achievement in a massive open online course. American Educational Research Journal, 52(5), 925-955. https://doi.org/10.3102/0002831215584621

Grund, C. K. (2015). How games and game elements facilitate learning and motivation: A literature review. Dans D. Cunningham, P. Hofstedt, K. Meer et I. Schmitt (dir.), Lecture Notes in Informatics (LNI) - Proceedings, Vol. P-246 (p. 1279-1293). Récupéré du site d'Augsburg University : http://www.wiwi.uni-augsburg.de

Guay, F., Ratelle, C. F., Roy, A. et Litalien, D. (2010). Academic self-concept, autonomous academic motivation, and academic achievement: Mediating and additive effects. Learning and Individual Differences, 20(6), 644-653. https://doi.org/10.1016/j.lindif.2010.08.001

Hew, K. F. et Cheung, W. S. (2014). Students' and instructors' use of massive open online courses (MOOCs): Motivations and challenges. Educational Research Review, 12, 45-58. https://doi.org/10.1016/j.edurev.2014.05.001

Ho, A. D., Reich, J., Nesterko, S., Seaton, D. T., Mullaney, T., Waldo, J. et Chuang, I. (2014). HarvardX and MITx: The first year of open online courses (HarvardX and MITx Working Paper No. 1). https://doi.org/10.2139/ssrn.2381263 
Jordan, K. (2015). Massive open online course completion rates revisited: Assessment, length and attrition. International Review of Research in Open and Distributed Learning, 16(3), 341358.

https://doi.org/10.19173/irrodl.v16i3.2112

Karsenti, T. (2013). MOOC : révolution ou simple effet de mode? Revue internationale des technologies en pédagogie universitaire, 10(2), 6-37. https://doi.org/10.7202/1035519ar

Karsenti, T. (2015). MOOCs: Facts and figures. International Journal of Technologies in Higher Education, 12(2), 154-158. https://doi.org/10.18162/ritpu-2015-v12n12-14

Karsenti, T., Depover, C., Komis, V. et Dumouchel, G. (2015). La pédagogie universitaire face aux MOOCs : compte-rendu d'expériences, tendances actuelles et futures. Revue internationale des technologies en pédagogie universitaire, 12(1-2), 7-8. https://doi.org/10.18162/ ritpu-2015-v12n12-01

Kerr, J., Houston, S. et Marks, L. (2015). Building and executing MOOCs: A practical review of Glasgow's first two MOOCs. Récupéré de http://www.openeducationeuropa.eu

Kizilcec, R. F. et Schneider, E. (2015). Motivation as a lens to understand online learners: Toward data-driven design with the OLEI scale. $A C M$ Transactions on Computer-Human Interaction, 22(2), 54-69. https://doi.org/10.1145/2699735

Konnikova, M. (2014, 7 novembre). Will MOOCs be Flukes? The New Yorker. Récupéré de http://www.newyorker.com

Krause, M., Mogalle, M., Pohl, H. et Williams, J. J. (2015, mars). A playful game changer: Fostering student retention in online education with social gamification. Dans Proceedings of the Second (2015) ACM Conference on Learning@Scale (p. 95-102). ACM. https://doi.org/10.1145/2724660.2724665

L'Écuyer, R. (1990). Méthodologie de l'analyse développementale de contenu : méthode GPS et concept de soi. Sillery, Canada : Presses de l'Université du Québec.

Miles, M. B. et Huberman, A. M. (2003). Analyse des données qualitatives. Bruxelles, Belgique: De Boeck Supérieur.
Morales, M., Amado-Salvatierra, H. R., Hernández, R., Pirker, J. et Gütl, C. (2016). A practical experience on the use of gamification in MOOC courses as a strategy to increase motivation. Dans L. Uden, D. Liberona et B. Feldmann (dir.), Learning Technology for Education in Cloud - The Changing Face of Education (p. 139-149). Cham, Suisse : Springer International Publishing. https://doi.org/10.1007/978-3-319-42147-6_12

OCDE. (2015). Connectés pour apprendre? Les élèves et les nouvelles technologies, série PISA. OCDE Publishing, Paris. Repéré à https:// www.oecd.org/fr/edu/scolaire/Connectes-pourapprendre-les-eleves-et-les-nouvelles-technologies-principaux-resultats.pdf

Pintrich, P. R. et Schunk, D. H. (2002). Motivation in education: Theory, research, and applications ( $2^{\mathrm{e}}$ éd.). Upper Saddle River, NJ : Merrill Prentice-Hall.

Ryan, R. M. et Deci, E. L. (2000). Self-determination theory and the facilitation of intrinsic motivation, social development, and well-being. American Psychologist, 55(1), 68-78. https://doi.org/10.1037//0003-066x.55.1.68

Schieb, L. J. et Karabenick, S. A. (2011). Teacher motivation and professional development: A guide to resources. Récupéré du site de Math and Science Partnership - Motivation Assessment Program : http://mspmap.org

Sharples, M., Kloos, C. D., Dimitriadis, Y., Garlatti, S. et Specht, M. (2015). Mobile and accessible learning for MOOCs. Journal of Interactive Media in Education, (1), 1-8. https://doi.org/10.5334/jime.ai

Xiong, Y., Li, H., Kornhaber, M. L., Suen, H. K., Pursel, B. et Goins, D. D. (2015). Examining the relations among student motivation, engagement, and retention in a MOOC: A structural equation modeling approach. Global Education Review, 2(3), 23-33. Récupéré de http://ger.mercy.edu

Yeager, C., Hurley-Dasgupta, B. et Bliss, C. A. (2013). cMOOCs and global learning: An authentic alternative. Journal of Asynchronous Learning Networks, 17(2), 133-147. Récupéré de http://eric.ed.gov 